$\#\left(\bigcup_{s \in \omega} A\left(K_{\alpha}^{\prime}, \alpha, s\right)\right)<c$ and $\exists p_{\alpha} \in C_{\alpha} \backslash \cup A\left(K_{\alpha}^{\prime}, \alpha, s\right)$. Let $K_{\alpha}=K_{\alpha}^{\prime}$ $\cup\left\{p_{\alpha}\right\}$. Then $K_{\alpha}$ fulfills the inductive assumptions and $K=\cup K_{\alpha}$ satisfies $\operatorname{dim} K^{s}=n-1$, for each $s \in \omega$. Hence, by Lemma 4, $\operatorname{dim} K^{\omega}<n$. But $K^{\omega} \supset K^{\prime}$ where $K^{\prime} \stackrel{T}{\approx} K$ and $\operatorname{dim} K \geqq n-1$. Thus $\operatorname{dim} K^{\omega}=n-1$.

iv a

\title{
REFERENCES
}

1. W. Hurewicz and H. Wallman, Dimension theory, Princeton Univ. Press, Princeton, N. J., 1941.

2. J. Nagata, Modern dimension theory, Interscience, New York, 1965.

Louisiana State University

\section{IMMERSIONS INTO MANIFOLDS OF CONSTANT NEGATIVE CURVATURE}

\section{EDSEL F. STIEL ${ }^{1}$}

1. Introduction. Let $M$ and $\bar{M}$ denote $C^{\infty}$ Riemannian manifolds, $K$ and $\bar{K}$ their respective sectional curvature functions, and $\psi: M \rightarrow \bar{M}$ an isometric immersion. A consequence of Theorem 2 of [5] is that if at any point $m \in M, K(\pi)<\bar{K}(d \psi(\pi))$, where $\pi$ is some plane in $M_{m}$, (the tangent space to $M$ at $m$ ) then there are no $\psi$ that immerse $M^{d}$ in $\bar{M}^{d+k}$ unless $k$ is greater than or equal to $d-1$. By restricting $M$ to be compact and $\bar{M}$ to be complete and simply connected, O'Neill has shown in [3] that there are no isometric immersions of $M^{d}$ in $\bar{M}^{d+k}$ when $K \leqq \bar{K} \leqq 0$ on $M$ unless $k$ is greater than or equal to $d$. Amaral (Theorem A of [1]) considered immersions of compact $M^{d}$ in $H^{d+1}(\bar{C})$, $(d+1)$-dimensional hyperbolic space of curvature $\bar{C}$, and by only assuming $K \leqq 0$ proved that there are no isometric immersions of $M^{d}$ in $H^{d+1}(\bar{C})$. Using methods similar to those of [3] we prove a theorem which strengthens O'Neill's result in the case that $\bar{M}$ is of constant negative curvature and includes Amaral's result.

\section{Results.}

TheOREM. Let $M$ be a compact d-dimensional Riemannian manifold and let $\bar{M}$ be a complete simply connected Riemannian manifold of constant curvature $\bar{C} \leqq 0$ and of dimension less than $2 d$. If the sectional

Received by the editors July 13, 1966.

${ }^{1}$ Part of the research in this paper was done while the author was the recipient of an NSF Research Participation award in Mathematics at the University of Oklahoma. 
curvature function of $M$ satisfies $K \leqq 0$, then $M$ cannot be isometrically immersed in $\bar{M}$.

There exist immersions of $H^{d}(C)$ in $H^{2 d-1}(\bar{C})$ for any $\bar{C}<C \leqq 0$ (where $H^{d}(0)=R^{d}, d$-dimensional Euclidean space); our theorem shows there are no compact ones. It is also known that the flat torus $T^{d}$ can be isometrically immersed in $H^{2 d}(\bar{C})$ for any $\bar{C}<0$; the theorem above shows a similar immersion of $T^{d}$ cannot be found in $H^{2 d-1}(\bar{C})$.

We express the information contained in the second fundamental form operators of the immersion $\psi$ by means of the operators $T_{x}: \bar{M}_{m} \rightarrow \bar{M}_{m}$ where $x \in M_{m}$ (see page 191 of [2]). The $T_{x}$ are skewsymmetric, bilinear, and interchange $d \psi\left(M_{m}\right)$ and the orthogonal complement of $d \psi\left(M_{m}\right)$ in $\bar{M}_{m}, M_{m}^{\perp}$. To simplify notation, we identify $M_{m}$ and $d \psi\left(M_{m}\right)$ when it seems safe to do so. In terms of these operators the Gauss equation for an immersion becomes $K(\pi)=\Delta_{x y}$ $+\bar{K}(d \psi(\pi))$ where $x$ and $y$ span $\pi$ and

$$
\Delta_{x y}=\left(\left\langle T_{x} x, T_{y} y\right\rangle-\left|T_{y} x\right|^{2}\right) /|x \wedge y|^{2} .
$$

The real valued function defined by $f(x)=\left|T_{x} x\right|$ for unit $x \in M_{m}$ has the following properties (see Lemmas 2 and 3 of [4]): If $y$ is a critical point of $f$ such that $f(y) \neq 0$, then $\left\langle T_{y} y, T_{y} x\right\rangle=0$ for all $x$ in $M_{m}$ that are perpendicular to $y$. Also $T_{y} x=0$ implies $\langle x, y\rangle=0$. If $y$ is a minimum of $f$ such that $f(y) \neq 0$ and $x$ is a unit vector orthogonal to $y$ then $\Delta_{x y}$ $+3\left|T_{y} x\right|^{2} \geqq\left|T_{y} y\right|{ }^{2}$. We shall also use the classical result that the curvature operators of the constant curvature manifold $\bar{M}(\bar{C})$ have the form $R_{x y} z=\bar{C}\{\langle x, z\rangle y-\langle y, z\rangle x\}$.

Proof of Theorem. Because of the result quoted above from [3], we need only consider the case in which $\bar{C}<0$. We assume an immersion $\psi: M^{d} \rightarrow \bar{M}^{d+k}(\bar{C})$ exists with $k<d$ and arrive at a contradiction. Let $\bar{m}$ be a fixed point in $\bar{M}$ and let $\psi(m) \in M$ be such that the distance from $\bar{m}$ to $\psi(m)$ is a maximum. Let $\sigma[0, b] \rightarrow \bar{M}$ be the unique unit speed geodesic from $\bar{m}$ to $\psi(m)$ and $z$ the velocity vector, $\sigma^{\prime}(b)$, of $\sigma$ at $\psi(m)$. For any unit $x$ in $d \psi\left(M_{m}\right)$ there exists a differentiable $r:[0, b]$ $\times[0,1] \rightarrow \bar{M}$ such that: (1) $r(\cdot, 0)=\sigma ;(2)$ for each $v \in[0,1], r(\cdot, v)$ is a geodesic; (3) $r(0, \cdot)=\bar{m}$ and $r(b, \cdot)$ is in $\psi(M)$; (4) if $X$ is the vector field on $\sigma$ such that $X(u)$ is the velocity of $r(u, \cdot)$ at $v=0$, then $X(0)=0$ and $X(b)=x$. Let $l(v)$ be the length of $r(\cdot, v)$; i.e. the distance from $\bar{m}$ to $r(b, v) \in \psi(M)$. Since $l(0)=0$ it follows that $z \in M_{m}^{\perp}$ and since 0 is a maximum of $l, l^{\prime \prime}(0) \leqq 0$. From the Synge formula for the second variation (cf. page 219 of [2]), we get

$$
l^{\prime \prime}(0)=\int_{0}^{b}\left\{\left|X^{\prime}(u)\right|^{2}-\bar{C}|X(u)|^{2}\right\} d u+\left\langle T_{x} x, z\right\rangle \leqq 0 .
$$


The $r(\cdot, v)$ curves are geodesics of $\bar{M}$ and therefore $X$ is a Jacobi field and satisfies the Jacobi equation $X^{\prime \prime}=R_{X \sigma^{\prime}}\left(\sigma^{\prime}\right)=\bar{C}\left(\left\langle X, \sigma^{\prime}\right\rangle \sigma^{\prime}\right.$ $\left.-\left\langle\sigma^{\prime}, \sigma^{\prime}\right\rangle X\right)=-\bar{C} X$, the last equality following since $\left\langle X, \sigma^{\prime}\right\rangle$ is constant along $\sigma$ and 0 at $b$. This implies that $X(u)=(\sinh c u) P(u)$, where $c=\sqrt{ }(-\bar{C}), P$ is a parallel field on $\sigma$, and $X(b)=(\sinh c b) P(b)=x$. The last relation implies that $|P(u)|=(\sinh c b)^{-1}$. Substituting these values for $X(u)$ and $|P(u)|$ into the Synge formula and integrating we find that $\left\langle T_{x} x, z\right\rangle \leqq-(c / 2)(\sinh 2 b c)\left(\sinh ^{2} b c\right)^{-1}$ $=-c \operatorname{coth} b c<-c$.

The function $f(x)=\left|T_{x} x\right|$ for unit $x \in M_{m}$ takes on a minimum at, say, $y$. This minimum, since $\left\langle T_{y} y, z\right\rangle<-c$, exceeds $c$. Also, since $k<d$, there exists a unit $x$ in $M_{m}$ such that $T_{y} x=0$. By the results quoted from [4] we must have that $\langle x, y\rangle=0$ and also that $\Delta_{x y}+3\left|T_{y} x\right|^{2}$ $\geqq\left|T_{y} y\right|^{2}$ or $\Delta_{x y}>c^{2}=|\bar{C}|$. However, $K=\Delta_{x y}+\bar{C}>|\bar{C}|+\bar{C}=0$. This contradicts the hypothesis that $K \leqq 0$ and hence the proof of the theorem is complete.

\section{REFERENCES}

1. L. H. Amaral, Hypersurfaces in non-euclidean spaces, Doctoral dissertation, University of California, Berkeley, 1964.

2. R. L. Bishop and R. J. Crittenden, Geometry of manifolds, Academic Press, New York, 1964.

3. B. O'Neill, Immersion of manifolds of nonpositive curvature, Proc. Amer. Math. Soc. 11 (1960), 132-134.

4. - Umbilics of constant curvature immersions, Duke Math. J. 32 (1955), 149-160.

5. T. Otsuki, On the existence of solutions of a system of quadratic equations and its geometrical application, Proc. Japan Acad. 29 (1953), 99-100.

California State College at Fullerton 